\title{
Editorial
}

Anaesthesist 2019.68 (Suppl 2):S79

https://doi.org/10.1007/s00101-019-0554-6

(c) Springer Medizin Verlag GmbH, ein Teil von Springer Nature 2019

Check for
updates

R. Rossaint ${ }^{1} \cdot$ B. Zwißler ${ }^{2}$

${ }^{1}$ Klinik für Anaesthesiologie, Universitätsklinikum Aachen, Aachen, Deutschland

${ }^{2}$ Klinik für Anaesthesiologie, Klinikum der Universität München, LMU München, München, Deutschland

\section{Facharzt-Training Anästhesiologie}

\section{Von „Fallvignetten“ zum E-Learning-Tool}

In diesem Sonderheft der Zeitschrift Der Anaesthesist erfolgt der Startschuss für ein ehrgeiziges Projekt mit dem Namen „Facharzt-Training Anästhesiologie“: Unter Leitung von Prof. Dr. A. Heller und PD Dr. G. Breuer entsteht ein LernTool für die Facharztprüfung „Anästhesiologie", das anhand von zahlreichen Patientenkonstellationen das Fach in seinen 4 Säulen Anästhesie, Intensiv-, Notfall- und Schmerzmedizin aufarbeitet.

Nahezu alle unsere heute in der Facharztausbildung befindlichen Kollegen haben sich mit einem solchen aus „Fallvignetten" bestehenden Tool auf ihr Staatsexamen vorbereitet. Somit ist dieses Format bekannt, vertraut und beliebt. Das Format greift das Konzept des problemorientierten Lernens auf, von dem wir wissen, dass die Lernerfolge pro Zeiteinheit höher und nachhaltiger als bei vergleichbarem Lesen eines konventionellen Lehrbuchs sind. Zwar wird man hin und wieder nicht auf die Systematik eines Lehrbuchs beim Nachschlagen oder Bearbeiten eines bestimmten Sachverhalts verzichten wollen und können. Doch entscheidend für die Lehrmethode von Standardwissen muss sein, wie wir Menschen am besten Lerninhalte aufnehmen.

Natürlich könnte man argumentieren, dass Lerninhalte noch besser in der Simulation vermittelt werden. Jedoch kann dieses Instrument nur gezielt

Aus Gründen der besseren Lesbarkeit wird in diesem Beitrag überwiegend das generische Maskulinum verwendet. Dies impliziert immer beide Formen, schließt also die weibliche Form mit ein. und vereinzelt eingesetzt werden, da hiermit bis heute extrem hohe Kosten verbunden sind, die eben nicht durch Krankenkassen, Ärztekammern oder sonstige Einrichtungen übernommen werden. Im Vergleich zur Simulation sind dagegen Patientenkonstellationen oder Fallvignetten in beliebiger Anzahl und Diversität mit nur geringem Aufwand zu kreieren. Durch gezielte Fragen und Antworten kann das erforderliche Wissen - hier das Facharztwissen Anästhesiologie - in beliebiger Tiefe problemorientiert vermittelt werden. Im vorliegenden Heft werden nunmehr in einem ersten Aufschlag 25 unterschiedliche Patientenkonstellationen und Themenfelder entsprechend aufgearbeitet.

\section{) Mithilfe von 25 Fallvignetten werden die 4 Säulen der Anäs- thesiologie problemorientiert erarbeitet}

Die Verantwortlichen streben in den kommenden Jahren an, weitere Sonderhefte mit ähnlichen „Lernfällen“ zu schaffen. In einem weiteren Schritt wird dann anhand der vorliegenden Texte ein E-Learning-Tool mit unterschiedlichsten thematischen Modulen generiert.

Es ist den verantwortlichen Kollegen zu wünschen, dass sie Durchhaltevermögen und einen langen Atem beweisen. Da hier eine Lücke in unserem Teaching zum Facharzt geschlossen wird, werden ihnen in jedem Fall die vielen in der Facharztweiterbildung befindlichen Kollegen danken.

Im Namen der Schriftleitung von Der Anaesthesist

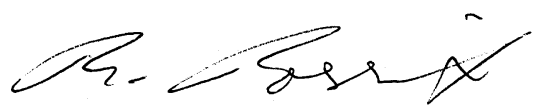

Prof. Dr. R. Rossaint

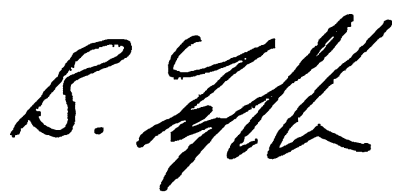

Prof. Dr. B. Zwißler

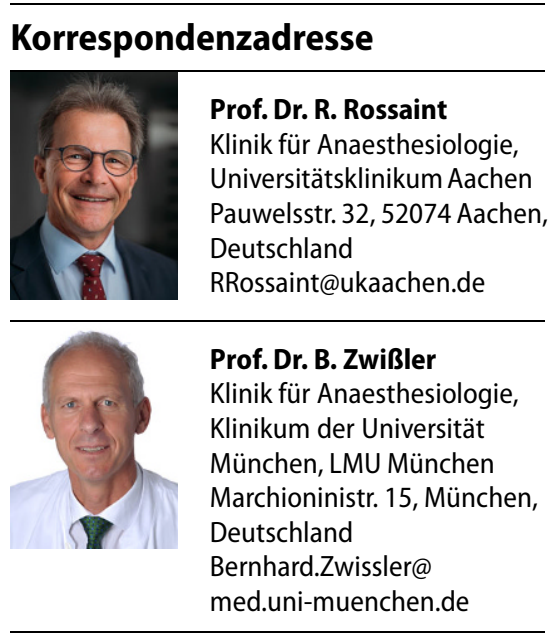

Interessenkonflikt. R. Rossaint und B. Zwißler geben an, dass kein Interessenkonflikt besteht.

The supplement containing this article is not sponsored by industry. 